Research

\title{
Research on college gymnastics teaching model based on multimedia image and image texture feature analysis
}

\author{
Ying $\mathrm{Wu}^{1}$. Jikun $\mathrm{Liu}^{2}$ \\ Received: 17 May 2021 / Accepted: 23 June 2021 \\ Published online: 01 July 2021 \\ (c) The Author(s) 2021 \\ OPEN
}

\begin{abstract}
With the rapid development of gymnastics technology, novel movements are also emerging. Due to the emergence of various complicated new movements, higher requirements are put forward for college gymnastics teaching. Therefore, it is necessary to combine the multimedia simulation technology to construct the human body rigid model and combine the image texture features to display the simulation image in texture form. In the study, GeBOD morphological database modeling was used to provide the data needed for the modeling of the whole-body human body of the joint and used for dynamics simulation. Simultaneously, in order to analyze and summarize the technical essentials of the innovative action, this experiment compared and analyzed the hem stage of the cross-headstand movement of the subject and the hem stage of the $180^{\circ}$ movement. Research shows that the method proposed in this paper has certain practical effects.
\end{abstract}

Keywords Multimedia image · Image texture · Feature analysis · Gymnastics teaching · Image capture

\section{Introduction}

At present, in domestic and foreign research, virtual human motion simulation technology is applied in the field of sports simulation, but there are relatively few software systems that can synthesize specific athletes' movements according to the requirements of coaches. Rhythmic gymnastics is a sport based on natural and rhythmic movements, accompanied by music or hand-held light instruments. It includes freehand gymnastics and light gymnastics (balls, rings, ropes, sticks, belts). The changes in the new rules of rhythmic gymnastics require new strategies for its development [1].

The technical analysis of the motion from the perspective of sports biomechanics is first of all the motion information collection and data processing analysis, followed by the study of human motion law [2]. The emergence of motion capture technology dates can back to the 1970s, and it is a high-tech device for accurately measuring the motion of moving objects in three-dimensional space. Based on computer graphics principles, it records the motion of a moving object (tracker) as an image through several video capture devices arranged in space. Then, it uses a computer to process the image data and obtain spatial coordinates $(X, Y, Z)$ of different objects (trackers) in units of time at different times [3]. Motion capture technology is widely used in many fields. In sports training, it can help the coach to observe the athlete's movement from different perspectives, and quantify the position, speed, acceleration and other data, so that the coach can correct the athlete's technical movements and greatly improve the athlete's training effect. In addition, it can monitor the entire sports training and competition process, and assist the referee decision-making referee [4].

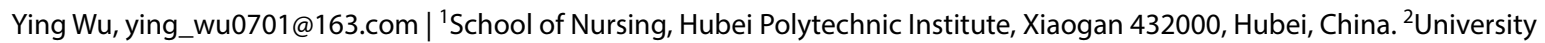
of Michigan, Ann Arbor 48104, USA. 
From a technical point of view, the essence of motion capture is to measure, track, and record the motion trajectory of an object in three-dimensional space. A typical motion capture device is generally composed of the following components: Sensor: A sensor is a tracking device that is fixed at a specific part of a moving object. It will provide the system with position information of the motion of the moving object, and it will generally determine the number of trackers as the level of detail is captured [5]. Signal capture device: It is responsible for capturing, recognizing, and responsibly transmitting motion data from a signal capture device to a computer system. Such devices vary depending on the type of system and they are responsible for the capture of position signals. For mechanical systems, it is a circuit board that captures electrical signals, and for optical systems, it is a high-resolution infrared camera [6]. Data transmission equipment: In particular, systems that require real-time effects require a large amount of motion data to be quickly and accurately transmitted from the signal capture device to the computer system for processing, and the data transmission device is used to accomplish this work. Data processing equipment: The data captured by the system needs to be corrected, and the 3D model must be combined to complete the computer animation production. These require us to apply data processing software or hardware to do the job. By means of the computer's high-speed computing power of data, the data can be processed, and the three-dimensional model can be moved naturally. Therefore, it is responsible for processing the original signal captured by the system, calculating the trajectory of the sensor, correcting and processing the data, and combining it with the three-dimensional character model [7]. So far, the commonly used motion capture technologies can be divided into mechanical, acoustic, electromagnetic and optical in principle [8]. Optical motion capture accomplishes the task of motion capture by monitoring and tracking specific spots on the target. In theory, for a point in space, as long as it can be seen by two cameras at the same time, according to the image and camera parameters taken by the two cameras at the same time, the position of the point in space at this moment can be determined [9]. When the camera shoots continuously at a high enough rate, the motion trajectory of the point is obtained from the image sequence [10]. The PTI we use is part of the optical motion capture technology and has the advantage of automatically defining the axes and coordinate points.

There are also many papers that introduce capture techniques conceptually or in terms of applications. Neng Dong and Zhiming Zhao introduced the composition and classification of capture technology and summarized and analyzed the application of motion capture technology in sports field and its prospects [11]. Combining with the reality of ethnic dances in Guizhou Province, Qun Cai and Jun Lu introduced the application of motion capture technology in minority dance art, and the basic production process of digital protection of dance art, and basically realized the digital protection of national dance [12]. However, in the specific research, the literature on the application of three motion capture techniques to study the technical movements of competitive sports has not been seen.

The modeling and simulation of gymnastics movements can be reproduced realistically and can observe the movements of athletes and the movement trajectories of each link in three dimensions, and output the movement parameters such as displacement, speed, force and moment of athletes at any time, which is convenient for quantitative analysis. By combining the principles of human physiology and physics [13] and researching ways to improve technical movements, physical training can be freed from the purely empirical state and enter the era of theoretical and digital. In addition, it is also possible to capture the movements of athletes with poor grades and compare them with the movements of elite athletes to help them train [14]. Based on the above analysis, this study starts with the simulation of gymnastics teaching in colleges and universities, and changes the traditional teaching methods, and analyzes the effectiveness of the proposed method.

\section{Introduction of digital simulation technology for multi-body dynamics}

Simulation technology is an indirect experimental technique. It mainly establishes a model similar to the research object or process, and indirectly studies the motion law of the object through the model. According to the type of model, it can be divided into: physical simulation, digital simulation. The multi-body dynamics simulation done in this paper belongs to the category of digital simulation. It is based on the multi-body system dynamics principle and establishes the required human dynamics digital model to simulate the human motion. Its advantages are low risk, high returns, and fast update [15].

Sports biomechanics usually regards the human body as a multi-body system, and the continuous development of multi-body system dynamics theory provides strong support for the structural design analysis and optimization of complex systems [16]. On this basis, the establishment of the dynamic equation of human motion and the acquisition of dynamic parameters in human motion are the key steps in the theoretical analysis of motion biomechanics, and it 
is one of the difficulties in the study of motion biomechanics. This step is often referred to as building a mathematical model, or simply referred to as modeling.

With the continuous improvement of the functions of computer simulation software, digital simulation combines traditional modeling methods with computer simulation. The virtual experiment conducted by the virtual model of the computer opens up a new way for the research and application of sports biomechanics.

MSC.ADAMS/LifeMod software was developed by the American Biomechanical Research Group (BRG) in collaboration with a number of orthopedic companies, sports equipment companies and clinical medical research centers. Moreover, it is based on the MSC.ADAMS system and is specifically designed for analysis of human dynamics software. Through the software, it is possible to construct an anatomical model from the local to the whole. The model can include subsystems such as bones, muscles, ligaments, and joints. The model can be driven by the muscles of various parts of the body, or driven by various control schemes, or by examining the motion and dynamic response of various parts of the body by applying external environmental influences [17].

The MSC.ADAMS/LifeMod software includes a variety of standard human models such as children, adults and models of different races. Moreover, the established mannequin can be easily coupled with any mechanical system established by MSC.ADAMS/View [18], so that the subsequent simulation analysis can accurately obtain the movement and internal force of the human body under any working conditions. At the same time, it allows us to deeply understand the mechanical characteristics behind the human body movements and the rules of motion skill control. In addition, it can simulate different situations according to the athlete's respective physiological characteristics, carry out optimization analysis, and then achieve the purpose of optimizing athletes' skills, and guide and assist sports training.

As shown in Fig. 1, the human body model established by MSC.ADAMS/LifeMod consists of 19 links, including the head, neck, upper torso, lower torso, pelvis, left and right shoulder blades, left and right upper arms, left and right forearms and hands, left and right thighs, left and right calves and feet. The links are connected by hinges of different degrees of freedom. The model has a total of 52 degrees of freedom. Among them, the left and right elbow/wrist/hip/ankle joint, the head-neck, the neck-upper torso, the upper-lower torso, and the lower torso-pelvis are connected by a ball joint of 3 degrees of freedom. The left and right shoulder/scapular joints are hinged by 2 degrees of freedom (rotating around the sagittal and coronal axes). The left and right knee joints are connected by a 1 degree of freedom column hinge (rotating only about the sagittal axis), and the other human body position and orientation have 6 degrees of freedom.

The establishment and algorithm of LIFEMOD software dynamics equation are as follows [19]:

The angular velocity of the rigid body $B_{i}$ is [20]:

$$
\omega_{i}=B_{i} \dot{p}_{i}
$$

In formula (1)

Fig. 1 LifeMod Human Body 19 Link Model (LifeMod Handbook, 2007)

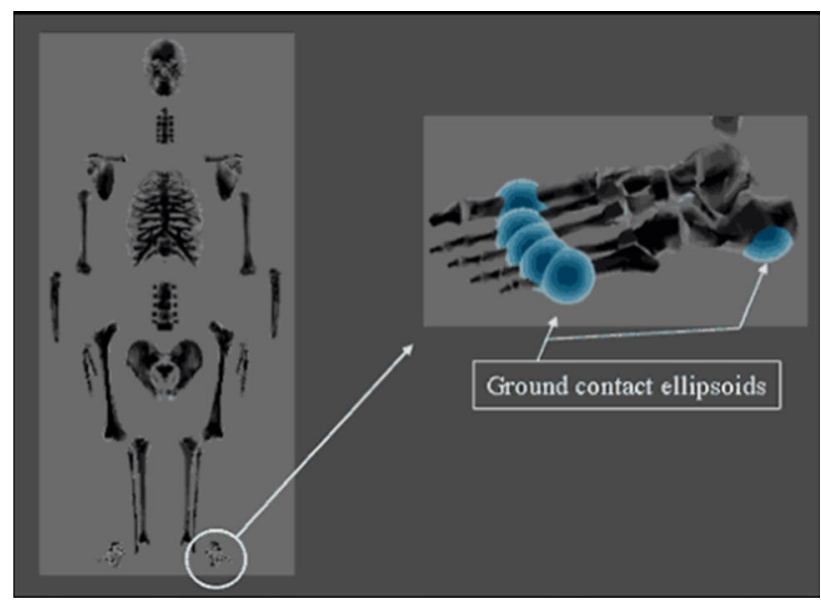




$$
B_{i}=\left[\begin{array}{ccc}
\sin \theta \sin \phi & 0 & \cos \theta \\
\cos \theta \sin \phi & 0 & \sin \theta \\
\cos \phi & 1 & 0
\end{array}\right]
$$

is the coordinate transformation matrix between the reference coordinate system of the rigid body centroid and the inertial system. The matrix form of the kinetic energy of the rigid body $B_{i}$ is:

$$
T_{i}=\frac{1}{2} \dot{r}_{i}^{T} m_{i} \dot{r}_{i}+\frac{1}{2} \omega_{i}^{T} J_{i} \omega_{i}^{T}
$$

In the formula (2), $\dot{r}_{i}$ is a rigid body centroid velocity, and $\vec{r}$ is a coordinate column in the inertial basis. $\omega_{i}$ is the coordinate array of the rigid body angular velocity $\vec{\omega}_{i}$ in its connected system, and $m_{i}$ is the $3 \times 3$ mass diagonal array of the rigid body, $J_{i}$ is the inertia tensor of the rigid body relative to the center of mass. By substituting Eq. (1) into Eq. (2), the expression of the Euler angle of kinetic energy is obtained [21]:

$$
T_{i}=\frac{1}{2} \dot{r}_{i}^{T} m_{i} \dot{r}_{i}+\frac{1}{2} \dot{p}_{i}^{T} B^{T} J_{i} B \dot{p}_{i}
$$

Each link is written with six Lagrangian equations corresponding to six generalized coordinates $q_{i}=\left(r_{i}^{T}, P_{i}^{T}\right)^{T}$ :

$$
\frac{d}{d t}\left[\begin{array}{l}
{\left[\frac{\partial T_{i}}{\partial \dot{r}_{i}}\right]^{T}} \\
{\left[\frac{\partial T_{i}}{\partial \dot{p}_{i}}\right]^{T}}
\end{array}\right]-\left[\begin{array}{l}
{\left[\frac{\partial T_{i}}{\partial r_{i}}\right]^{T}} \\
{\left[\frac{\partial T_{i}}{\partial p_{i}}\right]^{T}}
\end{array}\right]+\left[\begin{array}{c}
\Phi_{r_{i}}^{T} \lambda \\
\Phi_{p_{i}}^{T} \lambda
\end{array}\right]=\left[\begin{array}{c}
Q_{r_{i}} \\
Q_{p_{i}}
\end{array}\right] .
$$

In the formula (4), $\lambda=\left[\lambda_{1} \lambda_{2} \cdots \lambda_{6 \times 19}\right]^{T}$ is a Lagrangian multiplier array. $Q_{r_{i}}$ and $Q_{p_{i}}$ are the generalized force arrays corresponding to $r_{i}, p_{i}$, respectively. $\Phi_{r_{i}}^{T}$ and $\Phi_{p_{i}}^{T}$ are the partial derivative of the left part of the constraint equation to the generalized coordinates. The generalized angular momentum matrix is defined as [22]:

$$
\Gamma_{i}=\frac{\partial T_{i}}{\partial \dot{p}_{i}}=B_{i}^{T} J_{i} B_{i} \dot{p}_{i} .
$$

Since

$$
\frac{d}{d t}\left[\left[\frac{\partial T_{i}}{\partial \dot{r}_{i}}\right]^{T}\right]=m_{i} \ddot{r}_{i},\left[\frac{\partial T_{i}}{\partial r_{i}}\right]^{T}=0
$$

Therefore, Eq. (4) is transformed into:

$$
\left\{\begin{array}{c}
m_{i} \ddot{r}_{i}+\Phi_{r_{i}}^{T} \lambda=Q_{r_{i}} \\
\Gamma_{i}-\frac{\partial T_{i}}{\partial p_{i}}+\Phi_{p_{i}}^{T} \lambda=Q_{p_{i}}
\end{array}\right.
$$

A unified matrix form of the system dynamics equation is obtained by the 19 sets of equations generated in the 19 links:

$$
\left\{\begin{array}{c}
m \ddot{r}+\Phi_{r_{i}}^{T} \lambda-Q_{r_{i}}=0 \\
\dot{\Gamma}-\frac{\partial T}{\partial p}+\Phi_{p}^{T} \lambda-Q_{p}=0 \\
\Gamma-B^{T} J B \dot{p}=0
\end{array}\right.
$$

By integrating the above dynamic equations with the constraint equations, the complete algebra-differential equations of the system can be obtained. Then, by using the solver that comes with MSC.ADAMS/LifeMod software, the relevant kinetic parameters are obtained. The human body rigid model is shown in Fig. 2. 
Fig. 2 Human body rigid model

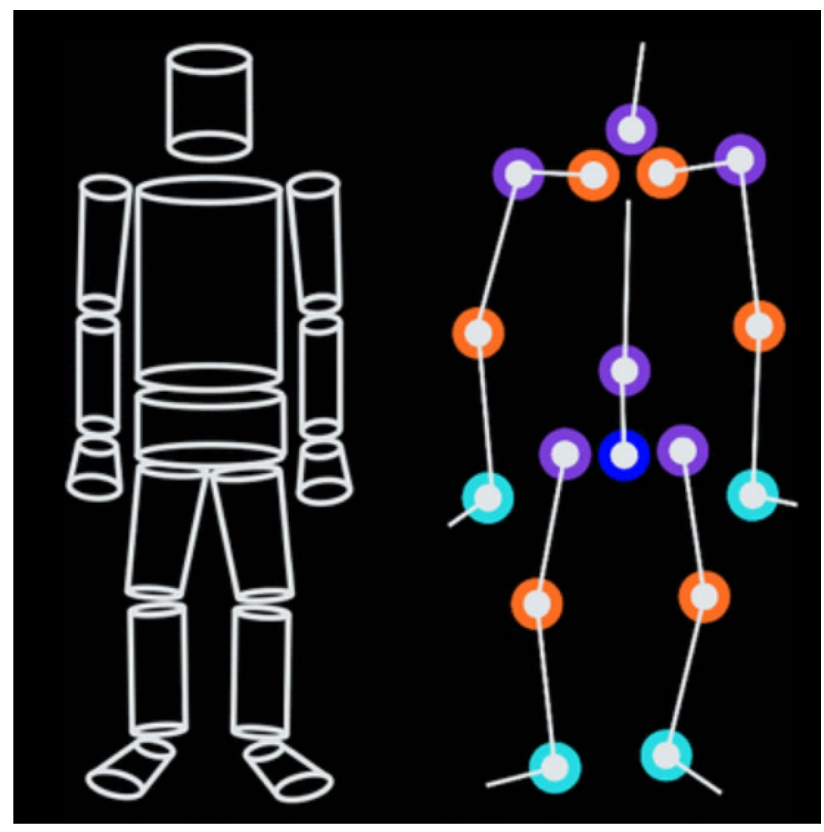

\section{Modeling and simulation process}

Next, the process of modeling and analysis of MSC.ADAMS/LifeMod will be described in detail with the example of the gymnastics movement model of "Scorpion swivel $180^{\circ}$ front hand flip + straight body front flip body $180^{\circ \prime}$, which is completed by Cheng Fei.

Step 1: The SLF interface file of the gymnastics mannequin is created.

Using the SIMI MOTION software, the three-dimensional motion coordinates of the human joint points at various times (time interval $0.004 \mathrm{~s}$ ) are obtained. The coordinates of the 14 joint points are included (as shown in Fig. 3 above):

Fig. 3 Human body model and joint selection

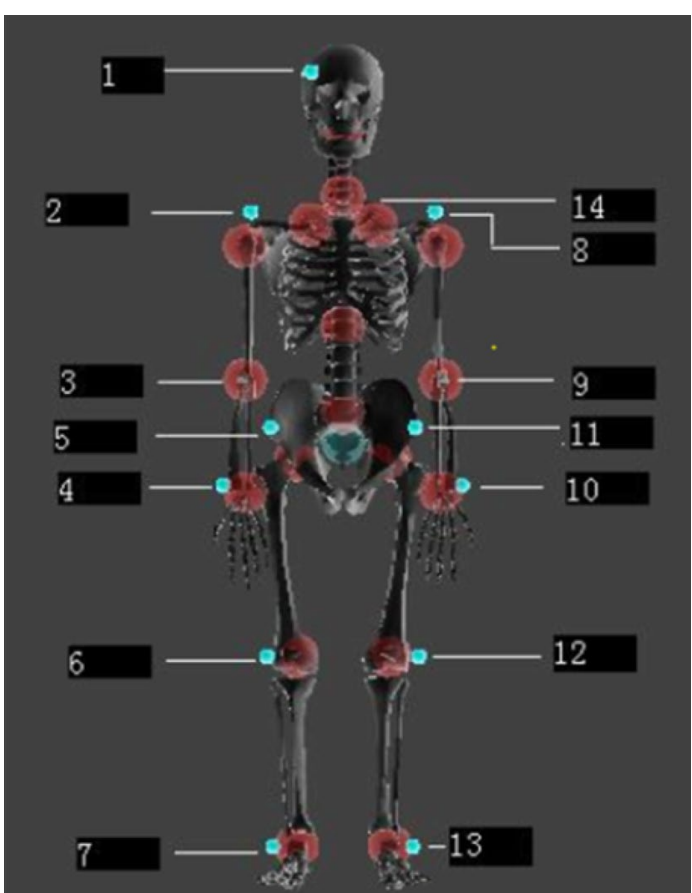


1. Head(RFHD): head $x$ head $y$ head $z$

2. Right shoulder(RSHO): right shoulder $x$ right shoulder $y$ right shoulder $z$

3. Right elbow(RELB): right elbow $x$ right elbow y right elbow $z$

4. Right wrist(RWRA): right wrist $x$ right wrist $y$ right wrist $z$

5. Right hip(RASI): right hip $x$ right hip $y$ right hip $z$

6. Right knee(RKNE): right knee $x$ right knee $y$ right knee $z$

7. Right ankle-bone(RANK): right ankle-bone $x$ right ankle-bone $y$ right ankle-bone $z$

8. Left shoulder(LSHO): left shoulder $x$ left shoulder $y$ left shoulder $z$

9. Left elbow(LELB): left elbow $x$ left elbow $y$ left elbow $z$

10. Left wrist(LWRA): left wrist $x$ left wrist $y$ left wrist $z$

11. Left hip(LASI): left hip $x$ left hip y left hip $z$

12. Left knee(LKNE): left knee $x$ left knee $y$ left knee $z$

13. Left ankle-bone(LANK): left ankle-bone $x$ left ankle-bone $y$ left ankle-bone $z$

14. Seventh cervical vertebra (C7): neck $x$ neck y neck $z$

Then, by using the Python scripting language, interface software suitable for human motion modeling and simulation is written. The purpose of the software is to convert the parameters such as mannequin name, gender, height, weight, age, status, and human morphology, human biomaterial, and the motion trajectory of the three-dimensional motion coordinates of the gymnastics analyzed in kinematics into an SLF format interface file that can be recognized by MSC. ADAMS/LifeMod software. The SLF format file for the human gymnastic model can be found in the appendix.

Step 2: Gymnastics mannequins, joints, and exercise pairs are established.

In this section, modeling is done through LifeMod's SLF interface file, which includes human body model parameters and motion trajectory data.

GeBOD (Generator of Body Data) morphological database was selected for modeling. The GeBOD Morphology Database provides the data needed to model the Joint Articulated Total Body (ATB) for dynamics simulation. GeBOD calculates the shape and mass properties of the body link as well as the position and kinematic properties of the joint. The human body model can be calculated based on gender, age, height, and weight, and a 19-section human body model can be established: Head, Neck, Upper-Torso, Central-torso, Lower-torso, right-Scapular, Right-Upper-Arm, Right-Lower-Arm, Right-Hand, Left-Scapular, Left- Upper-Arm,Left- Lower-Arm, Left-Hand, Right-Upper-Leg, Right-Lower-Leg, Right-Foot, Left-Upper-Leg, Left-Lower-Leg, Left-Foot. Each link has quality characteristics and direction.

After entering the operation interface of LifeMod,_Xchange is selected in the main menu, Import SLF model file is selected in the submenu, and SLF filename is specified to point to the SLF interface file that has been edited before modeling. Through the above steps, a complete initial skeleton model is established, and joints are established. At the same time, the three-dimensional coordinates of the human motion have also been introduced, that is, the motion pair of the model is established, as shown in Fig. 4 above.

Step 3: The initial pose and position of the model are adjusted.

First, the established human body model is rotated $180^{\circ}$ around the $Z$ axis. Then, in the main menu of LifeMod's operation interface, Posture is selected, and in the submenu, Configure Base Model is selected. At the same time, the angles of the joints in the sagittal, coronal and horizontal planes are adjusted to make the landmarks on the mannequin as close as possible to the three-dimensional coordinate points of the introduced initial human joint, as shown in Fig. 5 below.

Step 4: Balance analysis is carried out.

The purpose of running balance analysis is to bind the model to the position of the previously imported 3D coordinate data. After entering the analysis section, Analyze is selected in the main menu, and Dynamics is selected in the submenu. At the same time, the frozen motion pair is selected, the running time and time step are set, and then the balance analysis is performed, as shown in Fig. 6.

Step 5: Gymnastic equipment used in gymnastics was created—springboard, jumping platform, gymnastic mat.

In MSC.ADAMS/View, the modeling tool of the main toolbox is used to create a gymnastic environment, that is, a gymnastic instrument. The size and material properties of the gymnastic equipment created are based on the current national standards of the People's Republic of China and the requirements of the International Gymnastics Federation, and have been simplified to a certain extent on the basis of this, as shown in Fig. 7 below.

Step 6: The contact between the human body model and the environment was created.

The creation of the contact between the human body model and the environment means that the human body model is created in contact with the springboard, the jumping platform, and the gymnastics floor mat. After entering the contact 
Fig. 4 Human body model and initial guidance point

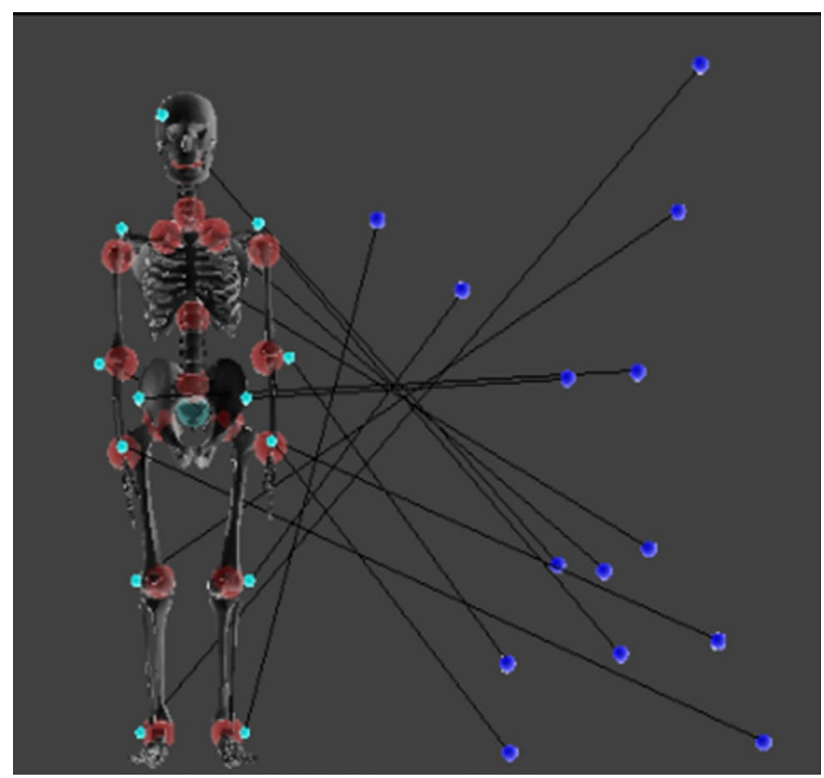

Fig. 5 Human body model after adjusting posture

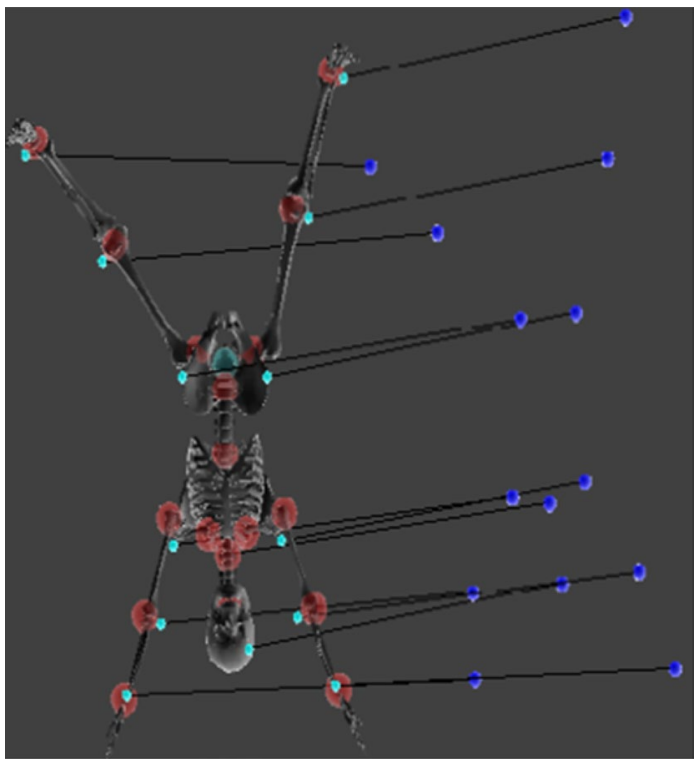

panel, Contacts is selected in the main menu. In the submenu, Creates individual contact is selected, and solid-solid is selected, that is, the contact between solid and solid. Then, the material parameters such as stiffness, damping and friction coefficient of the environment are set, and the contact between the feet and the springboard is established according to the three-dimensional coordinate data of the human body, and the contact between the hands and the jumping platform is established.

After that, in the sub-menu Create Base contact set, the Ellipsoid-plane is selected, that is, the ellipsoid is in contact with the plane. Then, according to the three-dimensional coordinate data of the human body, the contact between the feet and the gymnastic mat is established, and the material parameters such as stiffness, damping and friction coefficient of the gymnastic mat are set, as shown in Fig. 8 below.

Step 7: Inverse is run to perform inverse dynamics analysis.

In the previous step, the human body model was set in contact with three gymnastics instruments. In this paragraph, we perform three-stage simulation separately. Through the three-stage simulation analysis, we can see that the human body model will move along the previously introduced numerical trajectory in the motion pair. The difference between the given numerical trajectory and the trajectory displayed by the model during motion can be observed during the simulation. At the same time, all aspects of the human body are strictly in accordance with the assigned movement 
Fig. 6 Initial pose of the

human body model after bal-

ance analysis

Fig. 7 Environmental model

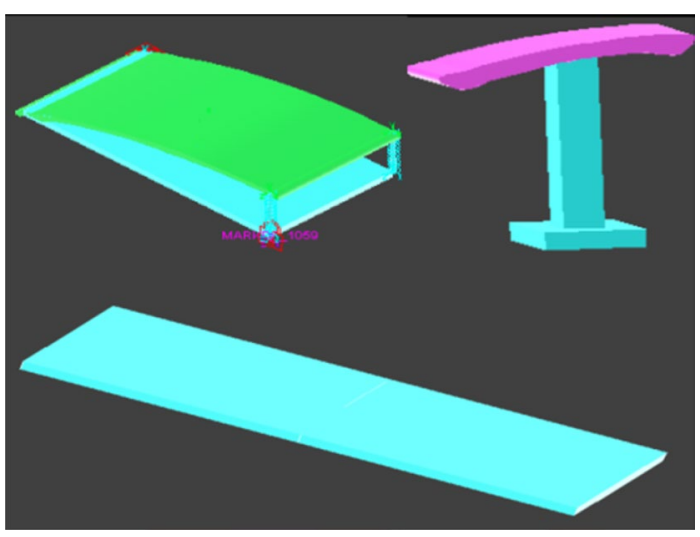

Fig. 8 Contact between the human body model and the environment

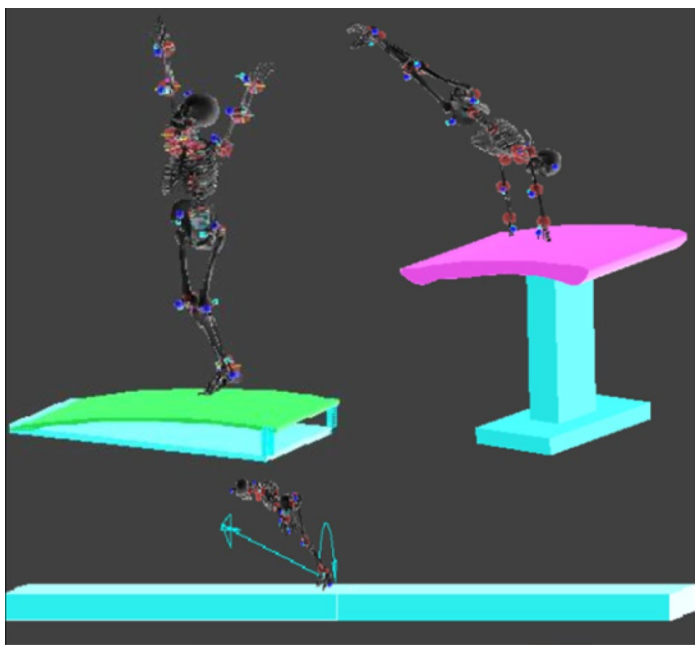

trajectory, and the marking point on the link is rigid attached to the model body. When the difference between the numerical value and the motion constraint occurs, it can be seen that the two small balls are separated, and the flexible connection between the two small balls allows a certain error in the assigned data, and the fault tolerance is within $0.1 \%$. In the reverse motion analysis process, the node unit records the process in preparation for the forward dynamics 
analysis to be performed. After entering the analysis section, we select Analyze in the main menu, select Dynamics in the submenu, set the gravity direction, running time and time step, and then perform the analysis.

Step 8: The forward dynamics analysis to be performed is prepared.

After recording the reversal analysis process, the Proportional-derivative controller is used to regenerate the run history, which includes removing the motion factor and updating the unit. At the same time, we also need to establish a Tracker Agent. The tracking factor is also a motion factor that uses the data recorded in the inverse dynamic simulation process to drive the model for forward dynamic simulation analysis. The joint node unit is updated by selecting Joints in the main menu and selecting Training in the submenu. Then, the trace agent is established by selecting Motion in the main menu and selecting Create Tracker Agent in the submenu.

Step 9: Forward is run to take forward dynamics analysis.

After entering the analysis section, we select Analyze in the main menu, select Dynamics in the submenu, and set the gravity direction, running time and time step. At the same time, Disable Motion Agents are selected, and the analysis is then performed. Finally, the results are processed in MSC.ADAMS/post.

\section{Experimental research}

In order to analyze and summarize the technical essentials of the innovative action, this experiment will compare the hem stage of the subject to do the cross-stand motion and the hem stage of the cross-stand up and $180^{\circ}$ action. The shooting step and the coordinate origin are selected as the image analysis crossover experiment. The height of the machine is $1.5 \mathrm{~m}$, one is facing the saddle horse sagittal plane, and its distance from the center of the pommel horse is $6 \mathrm{~m}$. The other machine is at an angle of $30^{\circ}$ to the sagittal plane, and the distance from the center of the pommel horse is $5 \mathrm{~m}$. The angle between the main axes of the two machines is $120^{\circ}$. After the shooting is completed, the video is imported into the computer for comparative analysis.

When the cross-handstand movement reaches the handstand movement, the maximum height of the center of gravity is $1.089 \mathrm{~m}$, and when the innovative movement reaches the handstand movement, the height of the center of gravity is $1.1035 \mathrm{~m}$. The reason is that the pommel horse rules require athletes not to have pauses and slow exertions while doing the action. Therefore, when the athletes cross the handstand, in order to prevent the excessive force from causing the athlete's center of gravity to move forward, most athletes do not pull the body up to the highest position and become straight upside down, so the center of gravity is lower. cross-handstand $+180^{\circ}$ movement requires the athlete to turn half a week under the premise of standing upside down. However, in order to successfully complete the movement, the athlete must find a way to keep the body stable when turning the body. Therefore, they can only make the inverted vertical to the vertical direction of the pommel horse to facilitate the turning, which leads to a higher center of gravity. The comparison table of the selection parameters of the hem stage of the cross-handstand action and the innovative action is shown in the following table.

It can be seen from Table 1 that the maximum center of gravity is $1.705 \mathrm{~m} / \mathrm{s}$ during the process of the hem of crosshandstand. In contrast, due to the higher center of gravity at the time of the handstand position, the speed of the downward swing of the innovative action is as large as $2.0614 \mathrm{~m} / \mathrm{s}$. This is attributed to the fact that the center of gravity of the cross-inversion plus $180^{\circ}$ starting position is higher, the gravitational potential energy is larger, and the swinging speed of the body's center of gravity is naturally faster during the hem of the body. Through the formula for calculating the moment, it can be obtained that the force exerted by the left-handed ring of the saddle horse on the supporting arms is greater than that when the crossed-inverted hem is completed.

Within a certain range, the change in the size of the shoulder angle can reflect the distance between the center of gravity of the human body and the support point of the pommel horse athlete during the hem (Fig. 9).

For these two movements, when the hem of the athlete's body reaches parallel with the pommel horse, the athlete's center of gravity is farthest from the support point. At this time, the smaller the shoulder angle, the closer the distance between the athlete's body center of gravity and the support point of the pommel horse. The shorter the torque, the smaller the gravity distance, the smaller the force of the support hand, and the easier the movement is. The shoulder angle of the cross-handstand movement is $61.805^{\circ}$, and the shoulder angle of the cross cross-handstand $+180^{\circ}$ rotation is $67.235^{\circ}$. A screenshot of the cross-handstand athletes reaching the level is shown in Fig. 10.

Shoulder angle and center of gravity speed are showed in Fig. 10 when the athlete releases his left hand and takes a single arm support when crossing the handstand. This moment is the moment when the athlete's action fails most. It is too early for the athlete to let go or the angle of the shoulder angle is too large to cause the support arm to fail to 
Fig. 9 Screenshot of the crosshandstand athletes reaching the horizontal moment

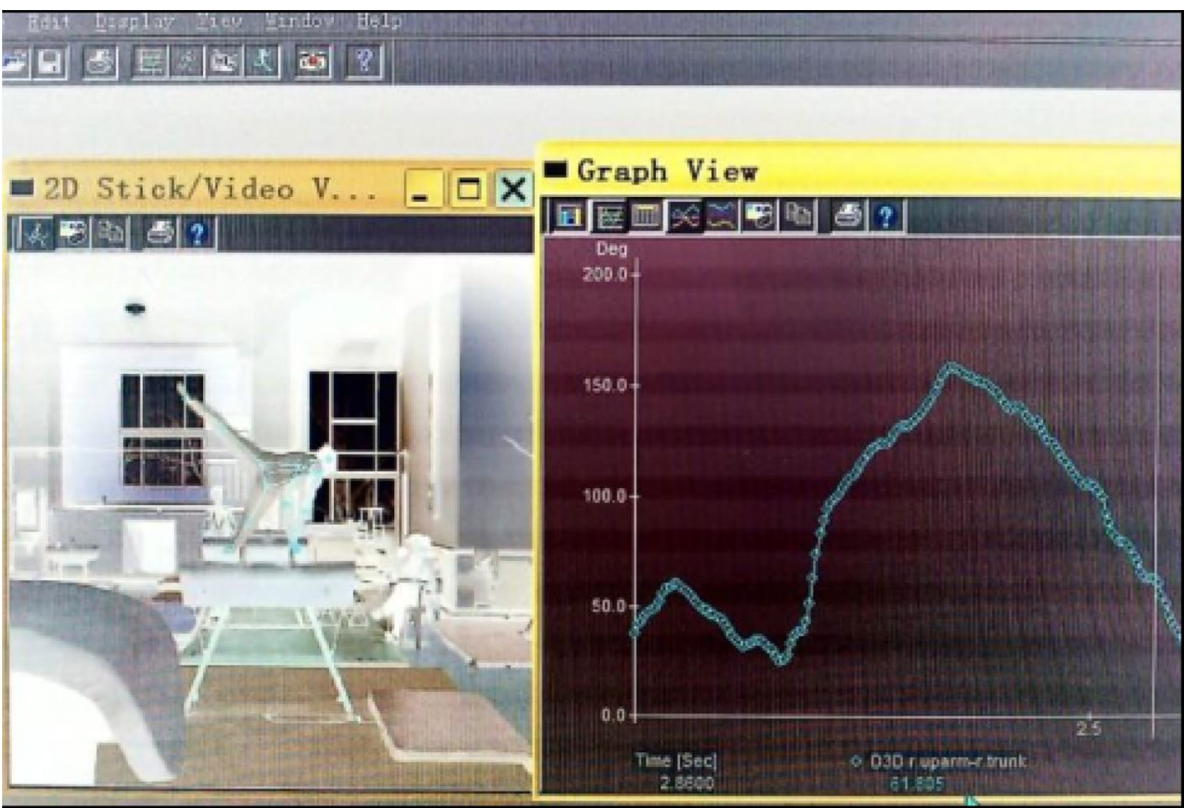

complete the support. A comparative study of the differences in the two action parameters at this time is critical to analyzing the two actions and finding the cause of the hem failure. It can be seen from the comparison of parameters: the shoulder angles of the cross-reverse action and the innovative action are $50.288^{\circ}$ and $53.065^{\circ}$ at the moment, and the center-of-gravity speeds are $1.1624 \mathrm{~m} / \mathrm{s}$ and $1.2248 \mathrm{~m} / \mathrm{s}$. It can be seen that the innovative action athlete puts the left hand into a single arm support too early, the shoulder angle is larger, and the body hem speed is higher than the hem stage of cross-handstand. At the same time, the force generated by the right-hand support alone cannot balance the function of the body's hem, causing the leg to ride across the saddle.

\section{Discussion}

There are errors in any measurement, and many errors are difficult to avoid in scientific research. Therefore, the computer simulation results of the gymnastics feature in this study also have errors. After analysis, the main causes of errors are as follows: Firstly, since this study simplifies the various aspects of the human body into rigid bodies and does not perform enough for the biological characteristics of the human body, it will inevitably produce certain errors.

Secondly, the inertial parameters of the human body used in each link are estimated using regression equations. In this study, human body modeling only considers the four factors of gender, age, height and weight, but ignores the differences between individuals, which also leads to errors. Thirdly, when the motion analysis software performs kinematics analysis, due to the particularity of the gymnastics competition, manual analysis of high-speed recording can only be performed, which will also cause certain human error. Finally, when filtering and smoothing data, some useful data may be lost, especially for some data peaks, which also produces some computational errors.

Therefore, gymnastics modeling and simulation should minimize errors. Firstly, good modeling and simulation methods must be used. Secondly, it is necessary to make more detailed measurements on the human body morphological indicators of the research subjects, such as head width and shoulder width, etc., to reduce the differences between individuals. Thirdly, according to the requirements, when using the analysis software to perform manual dot analysis on high-speed video, it is necessary to repeat the analysis of multiple times to reduce the manual dot error. Fourth, we need to use the appropriate numerical method to filter and smooth the data to reduce the calculation and error.

This study also attempts to simulate and design new actions through computer simulation experiments, so that not only can the existing actions be technically diagnosed, but also the unknown actions can be studied, the key technical points of the action can be proposed, and the training plan can be determined to make the research more forwardlooking. Of course, the research in this area is still in the trial stage, and there is still a lot of work to be done. This remains to be further studied. 


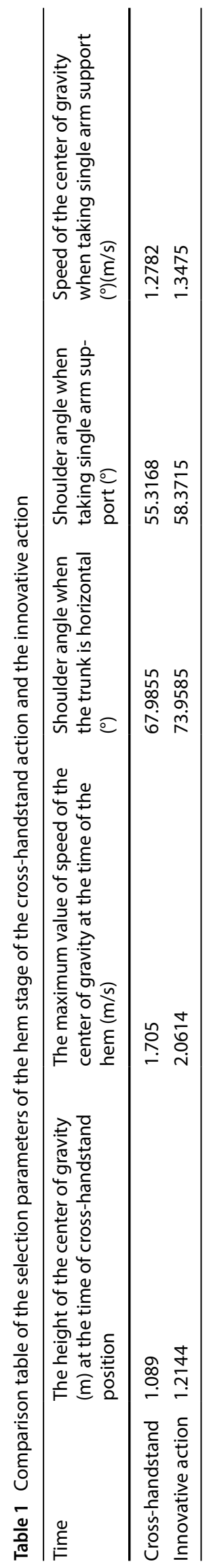


Fig. 10 Shoulder angle and center of gravity speed when the athlete releases his left hand and takes a single arm support when crossing the handstand

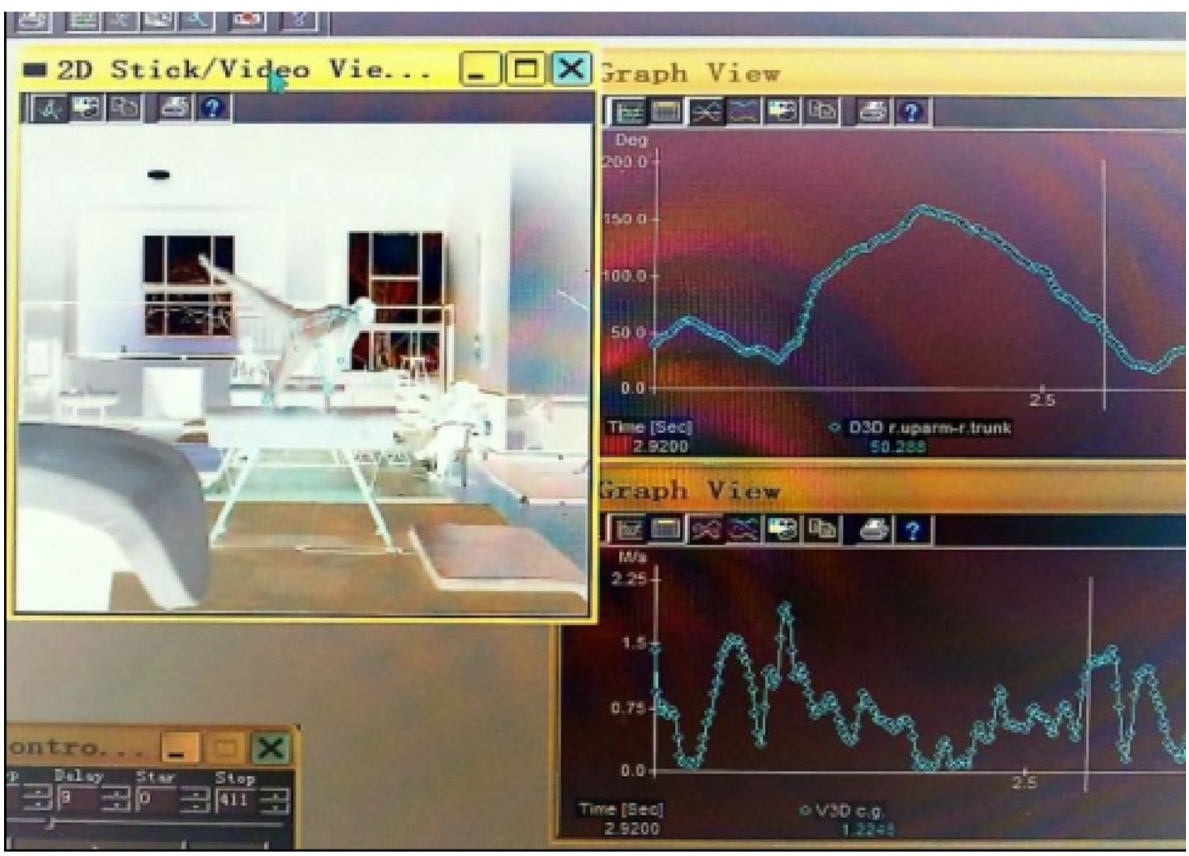

\section{Conclusion}

This paper mainly discusses how to use multimedia technology to realize the simulation of gymnastics teaching process in colleges and universities, and to achieve virtual demonstration of the deformation movement of the team to improve the efficiency of gymnastics teaching. In the gymnastics simulation study, according to the multisystem dynamics principle, this study establishes the required human dynamics model, and then uses the highspeed camera to photograph the gymnastics mobilizer's gymnastics movements and analyzes the film to obtain the kinematic parameters such as position and speed during the athlete's process. Then, computer simulation is carried out. Through the inverse dynamics simulation, the gymnastics movement process can be reproduced, and then the forward dynamics solution is performed according to the reverse simulation result, and the joint force and moment of each joint of the athlete during the movement can be obtained at any time. Through computer simulation, not only can the motion be analyzed according to the kinematic parameters such as position, speed, angular velocity, etc. when the athlete completes the action, but also a more comprehensive and accurate technical diagnosis can be made according to the dynamic data such as the timing of the athlete's joint force, the size of the force, and the time of the muscle working continuously, which can better guide the learning and training of the athlete.

Authors' contributions YW designed the research framework and wrote the manuscript, and JL was responsible for proofreading and optimization of the results. YW contributed significantly to analysis and manuscript preparation. JL helped perform the analysis with constructive discussions. All authors read and approved the final manuscript.

Data availability The data that support the findings of this study are available from Hubei Polytechnic Institute but restrictions apply to the availability of these data, which were used under license for the current study, and so are not publicly available. Data are however available from the authors upon reasonable request and with permission of Hubei Polytechnic Institute.

\section{Declarations}

Competing interests The authors declare no competing interests.

Open Access This article is licensed under a Creative Commons Attribution 4.0 International License, which permits use, sharing, adaptation, distribution and reproduction in any medium or format, as long as you give appropriate credit to the original author(s) and the source, 
provide a link to the Creative Commons licence, and indicate if changes were made. The images or other third party material in this article are included in the article's Creative Commons licence, unless indicated otherwise in a credit line to the material. If material is not included in the article's Creative Commons licence and your intended use is not permitted by statutory regulation or exceeds the permitted use, you will need to obtain permission directly from the copyright holder. To view a copy of this licence, visit http://creativecommons.org/licenses/by/4.0/.

\section{References}

1. Kirsch LP, Nadine D, Sumanapala DK, et al. Dance training shapes action perception and its neural implementation within the young and older adult brain. Neural Plast. 2018;2018:1-20.

2. Gingrasso S. Practical resources for dance educators! ndeo priorities for dance education: our call to action. Dance Educ Pract. 2017;3(1):6-11.

3. He Y, Zhang Q. Automatic generation algorithm analysis of dance movements based on music-action association. Cluster Comput. 2019;22:3553-61.

4. Ritchie LS. Dancing around the causal joint: challenging the theological turn in divine action theories. Zygon. 2017;52(2):361-79.

5. Dean SE, Nathanielsz J. An action of orientation - Skinner Releasing Technique in reflective practice. J Dance Somatic Pract. 2017;9(2):179-94.

6. Nota PMD, Chartrand JM, Gabriella R. Experience-dependent modulation of alpha and beta during action observation and motor imagery. BMC Neurosci. 2017;18:1.

7. Adair C, Burt R. Dance britannia: the impact of global shifts on dance in Britain. Congr Res Dance. 2018;2014:1-13.

8. Sedwick Caitlin. Up all night: BK channels' circadian dance with different calcium sources. J Gen Physiol. 2018;150(2):jgp:201711983.

9. Myers GE, Sparshott F. Off the ground: first steps to a philosophical consideration of dance. Res. 1992;52(1):243.

10. Han T, Yao H, Xu C, et al. Dancelets mining for video recommendation based on dance styles. IEEE Trans Multimedia. 2017;19(4):712-24.

11. Gibson CM. Dancing to the top: How collective action revitalized arts education in Boston. Arts Educ Pol Rev. 2017;119:217-30.

12. Kirsch LP, Cross ES. The influence of sensorimotor experience on the aesthetic evaluation of dance across the life span. Progr Brain Res. 2018;237:291-316.

13. Burzynska AZ, Karolina F, Taylor BK, et al. The dancing brain: structural and functional signatures of expert dance training. Front Human Neurosci. 2017;11:566.

14. Torregrosa-Salcedo E, Roig-Vila R, Blasco-Mira JE. Teaching Practice Workshop, a role-playing-based proposal for Higher Dance Studies. Res Dance Educ. 2017;18(1):90-106.

15. Young SN, Vanwye WR, Wallmann HW. Sport simulation as a form of implicit motor training in a geriatric athlete after stroke: a case report. Physiother Theory Pract. 2018;36(4):524-32.

16. Cameron PW, Soltero NC, Byers J. Effects of a 60 minute on ice game simulation on the balance error scoring system. Int J Exerc Sci. 2018;11(6):462-7.

17. Adam H, Jason T, Oestergaard NR, et al. Towards a complex systems approach in sports injury research: simulating running-related injury development with agent-based modelling. Br J Sports Med. 2018;53(9):560-9.

18. Narra N, Abe S, Dimitrov V, et al. Ricci-flow based conformal mapping of the proximal femur to identify exercise loading effects. Sci Rep. 2018;8(1):4823.

19. Karlinsky A, Zentgraf K, Hodges NJ. Action-skilled observation: Issues for the study of sport expertise and the brain. Prog Brain Res. 2017;234:263-89.

20. Patrascu M, Patrascu A, Beres I. An immune evolution mechanism for the study of stress factors in supervised and controlled systems. Evolving and adaptive intelligent systems (EAIS). Ljubljana: IEEE; 2017. p. 1-8. https://doi.org/10.1109/EAIS.2017.7954823.

21. Wychowanski M, Slugocki G, Orzechowski G, et al. Results of single sculling technique analysis using $1 \mathrm{~d}$ mathematical model. IFAC-Papers OnLine. 2018;51(2):879-83.

22. Forte $\mathrm{P}$, Marinho DA, Morais JE, et al. The variations on the aerodynamics of a world-ranked wheelchair sprinter in the key-moments of the stroke cycle: a numerical simulation analysis. PLoS ONE. 2018;13:2.

Publisher's Note Springer Nature remains neutral with regard to jurisdictional claims in published maps and institutional affiliations. 